\title{
Clinical Impact of Newly Diagnosed Right Bundle Branch Block (RBBB) in ST Segment Elevation Myocardial Infarction (STEMI)
}

\author{
Muhammad Firdani Ramadhan ${ }^{\mathrm{a}}$, Steve Freyssinet Karundenga, \\ I Gde Rurus Suryawan ${ }^{\mathrm{a}}$ \\ a drfirdani@gmail.com
}

${ }^{a}$ Department of Cardiology and Vascular Medicine, Faculty of Medicine, Universitas Airlangga - Dr. Soetomo General Hospital, Surabaya, 60286, Indonesia

\begin{abstract}
A newly diagnosed Right Bundle Branch Block is being investigated as a potential electrographic diagnostic for life-threatening STEMI equivalents (RBBB). RBBB was identified in 7.3 percent of individuals with NSTEMI and 9.1 percent of patients with STEMI. Previously, urgent angiography was suggested for patients with STEMI and Left Bundle Branch Block (LBBB), but it is now recommended for patients with STEMI and Right Bundle Branch Block (RBBB), as it is a poor prognostic sign of a higher mortality in STEMI patients. We report on a 67-year-old woman presented with a chief complaint of continuous heartburn since ten hours before admission to the hospital. Complaints of cold sweating and had comorbidities of diabetes mellitus, hypertension, and ischemic stroke 2 months ago. On arrival, a 12-lead electrocardiogram (ECG) revealed STEMI inferior with RBBB. The cardiac enzyme marker was increased due to an elevated serum troponin-I level of $10 \mathrm{ng} / \mathrm{mL}$ (cut off 0,01 ), an elevated serum creatinine level of $3.7 \mathrm{mg} / \mathrm{dL}$, leukocytosis (13850/L), and compensatory metabolic acidosis. She was treated with aspirin, clopidogrel, statins, ACE-i, and anticoagulants after being diagnosed with inferior STEMI with RBBB. As a practical matter of the COVID19 screening process, the patient did not require primary coronary intervention. The patient developed hemodynamic instability within two hours of being brought to the CVCU. The patient originally presented with transient ventricular tachycardia, which progressed to sustained ventricular tachycardia. Defibrillation with a dosage of 360 joules was used in conjunction with high-quality cardiopulmonary resuscitation until the patient was declared dead.
\end{abstract}

Keywords: Newly Diagnosed Right Bundle Branch Block; ST Segment Elevation Myocardial Infarction

\section{Introduction}

Myocardial infarction (MI) is described as the injury or death of the heart muscle caused by a blood supply restriction. STEMI is responsible for approximately $38 \%$ of all myocardial infarction cases in the United States. STEMI was defined as a new ST elevation at point $\mathrm{J}$ of at least 2 millimeters $(0.2$ millivolts $)$ in males and 1.5 millimeters ( 0.15 millivolts) in females in leads V2 and V3, and/or 1 millimeter ( 0.1 millivolts) in the other neighboring chest wall or limb leads. (Vasan and Benjamin 2016; Mozaffarian et al. 2016; Thygesen et al. 2012). A newly diagnosed Right Bundle Branch Block (RBBB) is being investigated as a potential electrographic diagnostic for life-threatening STEMI equivalents. RBBB was identified in 7.3 percent of individuals with NSTEMI and 9.1 percent of patients with STEMI (Figueroa-Triana et al. 2021).

Previously, urgent angiography was suggested for patients with STEMI and Left Bundle Branch Block (LBBB), but it is now recommended for patients with STEMI and Right Bundle Branch Block (RBBB), as it is a poor prognostic sign of a higher mortality in STEMI patients (Ibanez et al. 2018). Given the increased morbidity and mortality associated with newly diagnosed RBBB in STEMI patients, this article presents a literature review to assist physicians in comprehending the pathophysiology and rationale for managing newly diagnosed RBBB in STEMI patients. 


\section{Case Report}

A 67-year-old woman presented with a chief complaint of continuous heartburn since 10 hours before admission to the hospital. Complaints accompanied by cold sweat. The patient had a history of diabetes mellitus, hypertension, and ischemic stroke 2 months ago. Smoking history was denied. A physical examination revealed a heart rate of 101 beats per minute, a respiratory rate of 26 times per minute, and a blood pressure of $92 / 50 \mathrm{mmHg}$. On arrival, a 12-lead electrocardiogram (ECG) revealed a 100-beat-per-minute sinus rhythm with normal F and $\mathrm{H}$ axes, counter clockwise rotation (CCWR), an elevation of the ST segment in leads II, III, and aVF, and complete RBBB. (Figure 1).

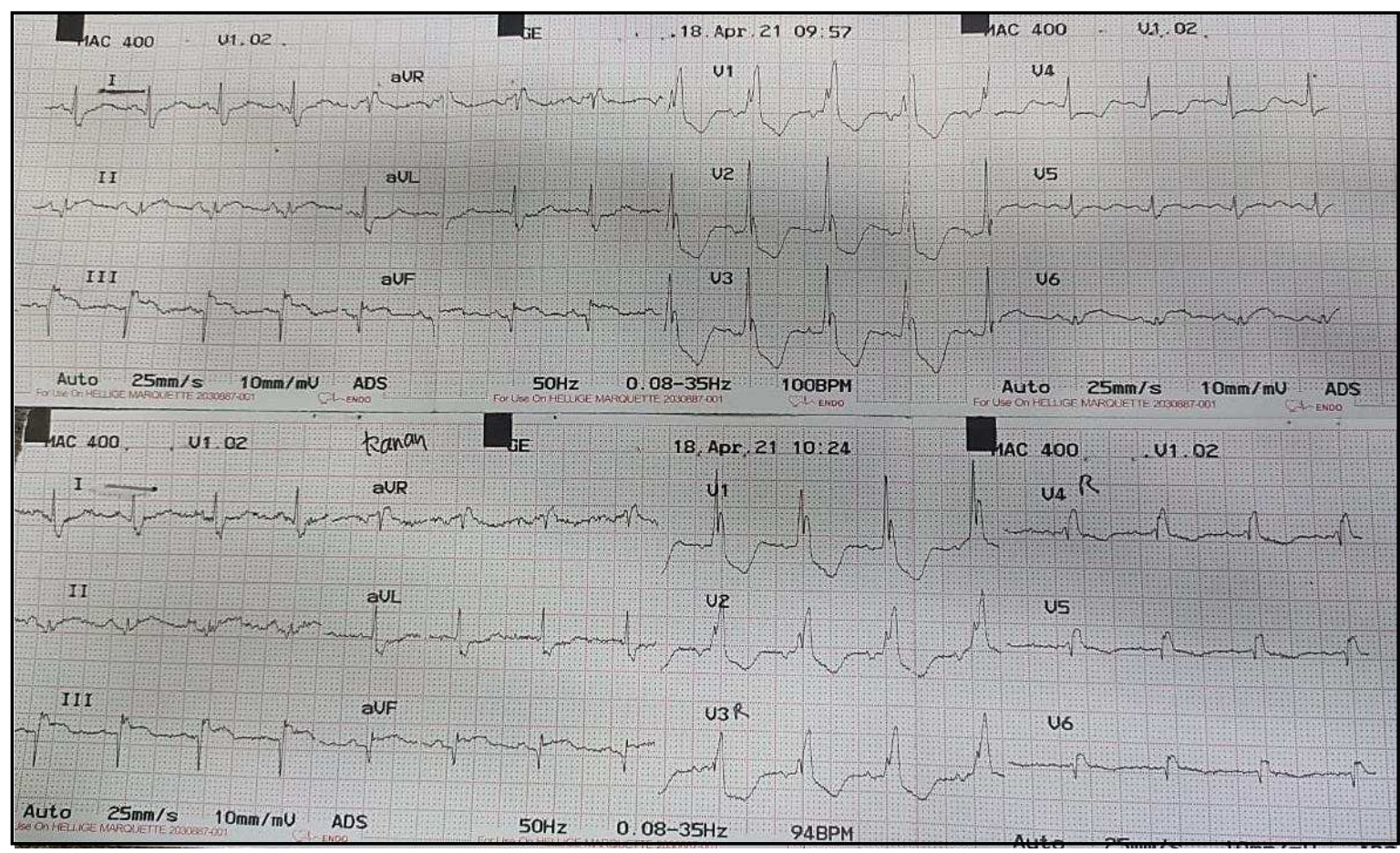

Figure 1. Electrocardiography showed sinus rhythm of 100 beats/min with normal $\mathrm{F}$ axis, normal $\mathrm{H}$ axis, counter clockwise rotation (CCWR), an elevation of the ST segment in leads II, III, and aVF, and complete RBBB.

On laboratory examination, the cardiac enzyme marker was elevated with serum troponin-I level $10 \mathrm{ng} / \mathrm{mL}$ (cut off 0,01$)$, elevated serum creatinine $(3.7 \mathrm{mg} / \mathrm{dL})$, leukocytosis $(13850 / \mathrm{L})$, and compensated metabolic acidosis. The chest X-ray showed cardiomegaly (Figure 2). Transthoracic echocardiogram (TTE) revealed inferior wall hypokinetics with a $47 \%$ ejection fraction (EF). Based on the diagnosis of Inferior STEMI with RBBB, he received aspirin, clopidogrel, statins, ACE-i and anticoagulants. Due to the COVID19 screening process, the patient did not undergo primary coronary intervention. 


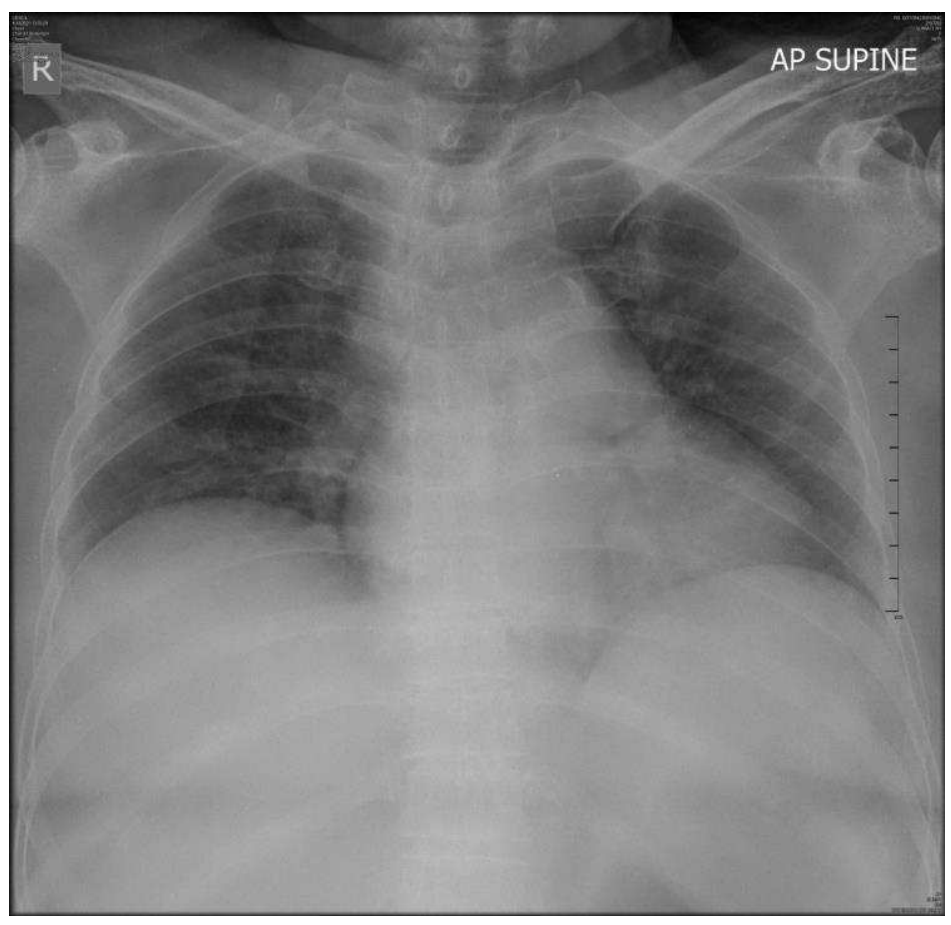

Figure 2. Chest X-Ray showed Cardiomegaly.

Within two hours of being transported to the CVCU, the patient was hemodynamically unstable. Syringe pump infusions of dobutamine and norepinephrine were used to correct the patient's hemodynamic status. The patient initially presented with non-sustained VT, which developed to sustained VT. Defibrillation with a dose of 360 joules and high-quality cardiopulmonary resuscitation were performed until the patient was declared dead.

\section{Discussion}

We present a case of newly diagnosed RBBB in an inferior STEMI patient. In addition, the patient is elderly and suffers from comorbid conditions such as diabetes mellitus, hypertension, and ischemic stroke 2 months ago. Herein, we discuss the characteristics and mechanism of ECG RBBB in STEMI, the pathophysiology of AMI-associated $\mathrm{RBBB}$, the clinical aspects of RBBB in AMI, and the rationale for managing newly diagnosed RBBB in STEMI patients.

RBBB affects the late phase of ventricular depolarization, causing RV depolarization to be delayed while left ventricular depolarization is unaffected, resulting in ECG abnormalities. The hallmark of the right bundle branch block is QRS duration $\geq 0,12$ seconds, rSR pattern in lead aVR and leads V1-V3 and S wave is wider than R wave or $>40$ $\mathrm{ms}$ in V5/V6 (Gussak et al. 2000).

In RBBB without AMI, the ST segment and $\mathrm{T}$ waves are typically discordant with the QRS complex's terminal component. This discordance is the consequence of the altered depolarization-induced repolarization sequence. Thus, a depressed ST segment with an inverted T wave due to an R' terminal wave appears in lead V1 when RBBB is present. In V6, the T wave is upright and the ST segment is elevated due to the presence of a terminal S wave. When RBBB complicates ST-elevation IMA, the ST segment becomes concordant with the QRS complex's terminal portion. 
ST-T changes occur independently of QRS changes, and are the major repolarization anomalies generated by ischemia (Gussak et al. 2000).

'Currents of injury' primarily mediated the mechanisms of RBBB with coronary occlusion. During the diastolic phase of myocardial infarction, the voltage gradient between the intact and damaged myocardium created currents of injury. It caused the ECG vector to be deflected downward, causing the ST segment appears elevated during the systolic phase. Because terminal R' waves are frequently present in these leads, the ST segment is elevated in V1 / V2 in anterior AMI. Similar changes can be seen in leads II, III, and AVF when an inferior myocardial infarction occurs (Gussak et al. 1999). When a complete RBBB pattern and a Q-wave infarct coexist, the criteria for the diagnosis of Q-wave AMI are the same as in patients with normal conduction due to the QRS complex will be abnormally wide (0.12 seconds or more), lead V1 will show a positive terminal deflection, and lead V6 will show a negative terminal deflection (wide S wave) (Ortega-Carnicer, Gómez-Grande, and Ambrós 2000; Shettigar et al. 2002).

There was $54.29 \%$ in the Left Anterior Descending artery (LAD) branch and $38.57 \%$ in the Right Circumflex artery (RCX) branch in the case of RBBB with Ischemic Related Artery (IRA). Both of the above percentages were significantly greater than those observed in non-BBBB IRA patients (Figueroa-Triana et al. 2021; Li et al. 2018). Because the right coronary artery serves the upper $90 \%$ of the interventricular septum, RCX obstruction can result in AV conduction abnormalties and bundle branch block. Decreased blood flow in the proximal RCA could result in right ventricle elongation and dilation, leading to the formation of new RBBB. Complete occlusion of the RCX may result in occlusion of the RV branch that originates from it (Pakbaz et al. 2013). Large areas of RV myocardial ischemia can cause the maximal rate of phase 0 depolarization to go down due to the lack of RV branch vasculature. As a consequence, the rate of conduction activation decelerates in the RV myocardium, resulting in asynchronous depolarization of the left and right ventricles, which might result in Major Adverse Cardiac Events (MACE) (Pakbaz et al. 2013; Li et al. 2018).

A total blockage of the proximal LAD or RCX is a typical of coronary AMI lesions with newly diagnosed RBBB. Obstruction of the RBB blood supply and ischemia or necrosis of a substantial portion of the RV myocardium are the features of AMI patients with newly identified RBBB (Kleemann et al. 2008). The massive infarct size found in AMI patients with RBBB is consistent with the high prevalence of three-vessel disease and left main disease identified in AMI patients with RBBB on presentation (Chan et al. 2016). Patients with RBBB are also more frequently reported to be older than those without RBBB and incidental finding of more comorbidities (Kleemann et al. 2008). Nevertheless, diffuse coronary artery disease in AMI patients with RBBB on the presentation may be an accidental finding rather than the prime cause (Farinha et al. 2021; Birnbaum et al. 2021).

The proportion of patients with TIMI 0/1 in IRA and those undergoing primary PCI was significantly greater in the RBBB group compared in the non-BBBB and LBBB groups (Li et al. 2018; Ibanez et al. 2018). The average length of stay, pro-BNP, left ventricular ejection fraction, heart failure, cardiac shock, cardiovascular mortality, and total MACE were significantly different in RBBB patients compared to non-BBB patients; Nevertheless, there was no significant difference between the two groups. (Abdel-Qadir et al. 2011; Aro et al. 2011; Barsheshet et al. 2011; Kleemann et al. 2008; $\mathrm{Li}$ et al. 2018). Thus, comparable to newly diagnosed LBBB, newly diagnosed RBBB is an independent predictor of in-hospital MACE and 30-days mortality (Wong et al. 2006). The probability of developing 
MACE was 4.682 times greater in individuals with AMI linked with the new RBBB than in persons without RBBB. The one-year survival rate was significantly different in RBBB patients. Furthermore, they had a worse survival rate than LBBB patients, a difference that was statistically significant (Farinha et al. 2021; Li et al. 2018; Ibanez et al. 2018).

While thrombolysis has the potential to significantly reduce mortality in AMI patients and has the advantages of high utility and ease of application, it also has a greater number of contraindications and a higher risk of bleeding, considering that STEMI patients with RBBB on presentation are older and have a greater number of comorbidities, as previously described in this discussion. As a result, STEMI patients with newly diagnosed RBBB had immediate early reperfusion. The goal is to keep the RBBB as short as possible and to revascularize the LAD and septal branch blood flow (Ibanez et al. 2018; Birnbaum et al. 2021).

\section{Conclusion}

Newly diagnosed RBBB on STEMI has a high prevalence of older patients, comorbidities, and diffuse coronary disease involvement. Hence, in guidelines for acute myocardial infarction, newly diagnosed RBBB should be regarded an indication for immediate revascularization. Understanding the characteristics, the pathophysiology, the clinical impact, and the rationale for managing RBBB in STEMI will assist doctors in making right decisions and will also help to reduce morbidity and mortality in this high-risk category of STEMI patients with RBBB on presentation.

\section{References}

Abdel-Qadir, Husam M, Jack V Tu, Peter C Austin, Julie T Wang, and Douglas S Lee. 2011. "Bundle Branch Block Patterns and Long-Term Outcomes in Heart Failure.” International Journal of Cardiology 146 (2): 213-18. https://doi.org/10.1016/j.ijcard.2010.01.012.

Aro, Aapo L, Olli Anttonen, Jani T Tikkanen, M Juhani Junttila, Tuomas Kerola, Harri A Rissanen, Antti Reunanen, and Heikki V Huikuri. 2011. "Intraventricular Conduction Delay in a Standard 12-Lead Electrocardiogram as a Predictor of Mortality in the General Population." Circulation. Arrhythmia and Electrophysiology 4 (5): 704-10. https://doi.org/10.1161/CIRCEP.111.963561.

Barsheshet, Alon, Ilan Goldenberg, Moshe Garty, Shmuel Gottlieb, Amir Sandach, Avishag Laish-Farkash, Michael Eldar, and Michael Glikson 2011. "Relation of Bundle Branch Block to Long-Term (Four-Year) Mortality in Hospitalized Patients with Systolic Heart Failure." The American Journal of Cardiology 107 (4): 540-44. https://doi.org/10.1016/j.amjcard.2010.10.007.

Birnbaum, Yochai, Kjell Nikus, Dan Atar, and Hani Jneid. 2021. “Is RBBB the New LBBB? Are We Going to Repeat the Same Mistakes?” Journal of Electrocardiology 65: 34-36. https://doi.org/10.1016/j.jelectrocard.2020.12.010.

Chan, William K, Shaun G Goodman, David Brieger, Keith A A Fox, Chris P Gale, Derek P Chew, Jacob A Udell, et al. 2016. "Clinical Characteristics, Management, and Outcomes of Acute Coronary Syndrome in Patients With Right Bundle Branch Block on Presentation." The American Journal of Cardiology 117 (5): 754-59. https://doi.org/10.1016/j.amjcard.2015.12.005.

Farinha, José Maria, Leonor Parreira, Rita Marinheiro, Marta Fonseca, Catarina Sá, Tatiana Duarte, Ana Esteves, Dinis Mesquita, Sara Gonçalves, and Rui Caria. 2021. "Right Bundle Brunch Block in Patients with Acute Myocardial Infarction Is Associated with a Higher In-Hospital Arrhythmic Risk and Mortality, and a Worse Prognosis after Discharge." Journal of Electrocardiology 64: 3-8. https://doi.org/https://doi.org/10.1016/j.jelectrocard.2020.11.007.

Figueroa-Triana, Juan F, Guillermo Mora-Pabón, Jerson Quitian-Moreno, Manuel Álvarez-Gaviria, Carolina Idrovo, Juan Sebastián Cabrera, José Alejandro Ramírez Peñuela, Yuldor Caballero, and Melissa Naranjo. 2021. "Acute Myocardial Infarction with Right Bundle Branch Block at Presentation: Prevalence and Mortality.” Journal of Electrocardiology 66: 38-42. https://doi.org/10.1016/j.jelectrocard.2021.02.009.

Gussak, I, R S Wright, P Bjerregaard, B R Chaitman, S H Zhou, S C Hammill, and S L Kopecky. 2000. "False-Negative and False-Positive ECG Diagnoses of Q Wave Myocardial Infarction in the Presence of Right Bundle-Branch Block." Cardiology 94 (3): 165-72. https://doi.org/10.1159/000047312.

Gussak, I, S H Zhou, P Rautaharju, P Bjerregaard, K Stocke, N Osada, Y Yokoyama, M Miller, S Islam, and B R Chaitman. 1999. "Right Bundle Branch Block as a Cause of False-Negative ECG Classification of Inferior Myocardial Infarction.” Journal of Electrocardiology 32 (3): 279-84. 
Ibanez, Borja, Stefan James, Stefan Agewall, Manuel J Antunes, Chiara Bucciarelli-Ducci, Héctor Bueno, Alida L P Caforio, et al. 2018. “2017 ESC Guidelines for the Management of Acute Myocardial Infarction in Patients Presenting with ST -Segment Elevation: The Task Force for the Management of Acute Myocardial Infarction in Patients Presenting with ST-Segment Elevation of the European Soci." European Heart Journal 39 (2): 119-77. https://doi.org/10.1093/eurheartj/ehx393.

Kazama, Itsuro, and Toshiyuki Nakajima. 2019. “Apparent ST Elevation in Right Bundle Branch Block Pseudo-Mimicking Myocardial Infarction.” SAGE Open Medical Case Reports. https://doi.org/10.1177/2050313X19827748.

Kleemann, Thomas, Claus Juenger, Anselm Kai Gitt, Rudolf Schiele, Steffen Schneider, Jochen Senges, Harald Darius, and Karlheinz Seidl. 2008. "Incidence and Clinical Impact of Right Bundle Branch Block in Patients with Acute Myocardial Infarction: ST Elevation Myocardial Infarction versus Non-ST Elevation Myocardial Infarction.” American Heart Journal 156 (2): 256-61. https://doi.org/https://doi.org/10.1016/j.ahj.2008.03.003.

Li, Jingchao, Xiaodong Li, Shujuan Dong, Yapan Yang, and Yingjie Chu. 2018. "Clinical Characteristics and Value in Early Reperfusion Therapy for New Onset Right Bundle Branch Block in Patients with Acute Myocardial Infarction.” Experimental and Therapeutic Medicine 15 (3): 2620-26. https://doi.org/10.3892/etm.2017.5661.

Mozaffarian, Dariush, Emelia J Benjamin, Alan S Go, Donna K Arnett, Michael J Blaha, Mary Cushman, Sandeep R Das, et al. 2016. "Heart Disease and Stroke Statistics-2016 Update: A Report From the American Heart Association." Circulation 133 (4): e38-360. https://doi.org/10.1161/CIR.0000000000000350.

Ortega-Carnicer, J, M L Gómez-Grande, and A Ambrós. 2000. "Right Bundle Branch Block-Induced Q Waves Simulating Anterior Myocardial Infarction Extension.” Journal of Electrocardiology 33 (4): 387-91. https://doi.org/10.1054/jelc.2000.9647.

Pakbaz, Marziyeh, Ali Kazemisaeid, Ahmad Yaminisharif, Gholamreza Davoodi, Masoumeh Lotfi Tokaldany, and Elham Hakki. 2013. "Coronary Anatomy Characteristics in Patients with Isolated Right Bundle Branch Block versus Subjects with Normal Surface Electrocardiogram." Journal of Cardiovascular Disease Research 4 (1): 47-50. https://doi.org/https://doi.org/10.1016/j.jcdr.2013.02.012.

Shettigar, Udipi R, Anu Pannuri, George H Barbier, and Doreen O Appunn. 2002. "Significance of Anterior Q Waves in Left Anterior Fascicular Block--a Clinical and Noninvasive Assessment." Clinical Cardiology 25 (1): 19-22. https://doi.org/10.1002/clc.4950250106.

Thygesen, Kristian, Joseph S Alpert, Allan S Jaffe, Maarten L Simoons, Bernard R Chaitman, Harvey D White, Hugo A Katus, et al. 2012. "Third Universal Definition of Myocardial Infarction." Circulation 126 (16): 2020-35. https://doi.org/10.1161/CIR.0b013e31826e1058.

Vasan, Ramachandran S, and Emelia J Benjamin. 2016. “The Future of Cardiovascular Epidemiology.” Circulation 133 (25): $2626-33$. https://doi.org/10.1161/CIRCULATIONAHA.116.023528.

Wong, Cheuk-Kit, Ralph A H Stewart, Wanzhen Gao, John K French, Christopher Raffel, and Harvey D White. 2006. "Prognostic Differences between Different Types of Bundle Branch Block during the Early Phase of Acute Myocardial Infarction: Insights from the Hirulog and Early Reperfusion or Occlusion (HERO)-2 Trial.” European Heart Journal 27 (1): 21-28. https://doi.org/10.1093/eurheartj/ehi622. 